\title{
The Expression of Social Dominance Following Neonatal Lesions of the Amygdala or Hippocampus in Rhesus Monkeys (Macaca mulatta)
}

\author{
M. D. Bauman, J. E. Toscano, and W. A. Mason \\ University of California, Davis
}

\author{
P. Lavenex \\ University of California, Davis, and Université de Fribourg
}

\author{
D. G. Amaral \\ University of California, Davis
}

\begin{abstract}
As part of ongoing studies on the neurobiology of socioemotional behavior in the nonhuman primate, the authors examined the social dominance hierarchy of juvenile macaque monkeys (Macaca mulatta) that received bilateral ibotenic acid lesions of the amygdala or the hippocampus or a sham surgical procedure at 2 weeks of age. The subjects were reared by their mothers with daily access to large social groups. Behavioral observations were conducted while monkeys were given access to a limited preferred food. This testing situation reliably elicited numerous species-typical dominance behaviors. All subjects were motivated to retrieve the food when tested individually. However, when a group of 6 monkeys was given access to only 1 container of the preferred food, the amygdala-lesioned monkeys had less frequent initial access to the food, had longer latencies to obtain the food, and demonstrated fewer species-typical aggressive behaviors. They were thus lower ranking on all indices of social dominance. The authors discuss these findings in relation to the role of the amygdala in the establishment of social rank and the regulation of aggression and fear.
\end{abstract}

Keywords: amygdaloid complex, social behavior, fear, aggression, macaque monkey

The primate amygdala has historically been implicated in a variety of behaviors associated with species-typical social behavior (Kling, 1992). Recent evidence indicates that selective damage to the amygdala does not disrupt fundamental components of social behavior, such as the ability to produce and respond to species-typical social signals and the ability to interact in a social context. However, bilateral amygdala lesions do alter several facets of social interactions, such as affiliation, aggression, and fear

M. D. Bauman and D. G. Amaral, Department of Psychiatry and Behavioral Sciences, Center for Neuroscience, California National Primate Research Center, and The M.I.N.D. Institute, University of California, Davis; J. E. Toscano, Department of Psychiatry and Behavioral Sciences, Center for Neuroscience, and California National Primate Research Center, University of California, Davis; W. A. Mason, Department of Psychology and California National Primate Research Center, University of California, Davis; P. Lavenex, Department of Psychiatry and Behavioral Sciences, Center for Neuroscience, California National Primate Research Center, and The M.I.N.D. Institute, University of California, Davis, and Institut de Physiologie, Université de Fribourg, Fribourg, Switzerland.

This research was supported by National Institute of Mental Health Grant R37MH57502 and by the base grant (RR00169) of the California National Primate Research Center (CNPRC). This work was also supported through the Early Experience and Brain Development Network of the MacArthur Foundation. We thank the veterinary and husbandry staff of the CNPRC for excellent care of the animal subjects. We also thank Jeffrey Bennett and Pamela Tennant for assistance with surgical procedures and Melissa Marcucci for assistance with behavioral data collection.

Corresponding concerning this article should be addressed to D. G. Amaral, The M.I.N.D. Institute, University of California, Davis, 2825 50th Street, Sacramento, CA 95817. E-mail: dgamaral@ucdavis.edu behaviors (Bauman, Lavenex, Mason, Capitanio, \& Amaral, 2004a, 2004b; Emery et al., 2001; Prather et al., 2001). Thus, the amygdala may play a role in complex social interactions that depend on the ability to correctly regulate aggression, affiliation, and fear responses. These behaviors are essential for the formation and maintenance of a social dominance hierarchy (Sade, 1967).

Most species of macaques demonstrate well-defined dominance hierarchies in both free-ranging (Drickamer, 1975) and captive social groups (Bernstein \& Mason, 1963; Janus, 1992). Dominance relationships among primates are defined by the history of previous agonistic encounters. The pattern of previous encounters allows accurate prediction of future interactions between two or more individuals (Bernstein, 1981). From an ethological perspective, an animal's ability to recognize social attributes and predict the outcome of a social encounter undoubtedly serves an adaptive function of minimizing injuries that might be sustained through aggressive conflicts (Bernstein, 1981). Indeed, macaques have developed a sophisticated repertoire of social signals that can be used to define and reinforce dominance relationships (Altmann, 1967; Missakian, 1972; Sade, 1967). These signals presumably evolved as an adaptation for successfully living within a social group (Cheney, Seyfarth, \& Smuts, 1986).

It is likely that several brain systems are involved in regulating the production and interpretation of species-typical social signals, including appropriate dominant and subordinate responses (Reader \& Laland, 2002). In the primate, substantial attention has been directed to the amygdala, because its damage leads to clear changes in dominance status. Rosvold, Mirsky, and Pribram (1954) first evaluated the role of the amygdala in social rank by lesioning the amygdalae of the 3 highest ranking male members of 
a small social group of macaques. Following amygdalectomy, 2 of the 3 previously high-ranking monkeys became submissive and fell to the bottom of the dominance hierarchy, whereas the 3rd monkey became abnormally aggressive. Similar decreases in social dominance following bilateral amygdala damage have been reported in other primate species (Kling, 1992; Kling \& Cornell, 1971; Plotnik, 1968). The underlying causes of the fall in dominance remain unclear. However, several possible explanations have been proposed, including social disinterest, rejection by other group members, and/or an inability to correctly produce and interpret social signals.

Though amygdala damage may play a role in maintaining social dominance in adult subjects, it is not known what effect neonatal lesions may have on the initial formation of species-typical dominance hierarchies in younger subjects. Previous research on neonatal amygdala lesions has suggested that early damage to the amygdala may have enduring consequences on social development, though methodological issues, such as rearing conditions and lesion techniques, may complicate the interpretations of behavioral data (Bachevalier, 1994; Prather et al., 2001; Thompson, Schwartzbaum, \& Harlow, 1969). Thompson and colleagues first reported that individually reared female rhesus monkeys who had sustained bilateral amygdala lesions early in life were abnormally fearful of conspecifics during the first year of development and abnormally subordinate to control subjects when observed at 3 and 6 years of age (Thompson, Bergland, \& Towfighi, 1977; Thompson et al., 1969). However, it is not known whether the abnormal subordinate behavior associated with early amygdala damage would be observed in a more naturalistic social-rearing environment, as differences in rearing conditions have long-lasting effects on the acquisition of dominance rank in rhesus monkeys (Bastian, Sponberg, Suomi, \& Higley, 2003; Mason, 1961).

As part of our ongoing evaluation of the role of the amygdala in the development of social behavior, we have examined the social dominance hierarchy of juvenile rhesus monkeys that had received bilateral lesions of either the amygdala or hippocampus or a sham procedure at 2 weeks of age. Our lab has previously reported that these neonatally amygdala-lesioned monkeys developed the fundamental components of social behavior (Bauman et al., 2004b). It is unknown whether early amygdala damage may alter more sophisticated social interactions, such as the establishment of social rank. To evaluate the role of the amygdala in dominancerelated behaviors, we provided the juvenile groups (mean age of 18 months) with a limited resource (i.e., a preferred food) that commonly induces agonistic behaviors among macaque monkeys (Southwick, 1967). Preferential access to food is an indication of social dominance, as the most dominant members of a group will generally gain initial access to a restricted food resource (Belzung \& Anderson, 1986). We predicted that if the amygdala is involved in the regulation of species-typical dominance behaviors, we would observe less access to the food, indicating lower social rank among the amygdala-lesioned subjects.

\section{Method}

All experimental procedures were developed in consultation with the veterinary staff at the California National Primate Research Center. All protocols were approved by the University of California, Davis, Institutional Animal Care and Use Committee.

\section{Subjects and Living Conditions}

Twenty-four infant rhesus monkeys (Macaca mulatta) naturally born of multiparous mothers were randomly assigned to one of three lesion conditions: bilateral amygdala lesions (5 females, 3 males), bilateral hippocampus lesions ( 5 females, 3 males), or sham-operated controls (4 females, 4 males). All surgeries were performed 12-16 days after birth. The infants were returned to their mothers following surgery and provided daily access to a socialization group consisting of 6 mother-infant pairs and 1 adult male that interacted for a minimum of $3 \mathrm{hr}$ per day, 5 days per week. The four socialization groups were each composed of 2 amygdalalesioned infants and their mothers, 2 hippocampus-lesioned infants and their mothers, and 2 sham-operated infants and their mothers. The age range between the youngest and oldest infant within each group was approximately 2 months. Three of the socialization groups were composed of 1 male and 1 female per lesion condition, and the fourth cohort consisted of 2 female amygdala-lesioned infants, 2 female hippocampus-lesioned infants, and 1 male and 1 female sham-operated infant.

When the youngest subject within a socialization group reached 6 months of age, the infants were permanently separated from their mothers but otherwise continued to experience the same housing and group socialization in the absence of their mothers. At this time, a new adult female was added to each socialization cohort to provide ongoing exemplars of adult female social behavior. At approximately 1 year of age, subjects became permanently socially housed ( $24 \mathrm{hr}$ per day) with their original socialization cohort in a chain-link enclosure $(2.13-\mathrm{m}$ width $\times 3.35$-m diameter $\times$ 2.44-m height). The mean age of subjects at the start of dominance testing was 1 year and 6 months, and their mean weight was $2.91 \pm 0.348 \mathrm{~kg}$. There was no significant difference in age and weight between experimental groups prior to the first and second phases of testing. Subjects had lived in their permanent social cohorts for approximately 5 months prior to the first phase of dominance testing.

It is important to note that one of the original male amygdala-lesioned subjects died at approximately 1 year of age because of health reasons unrelated to the lesion procedure and was replaced with an alternative neonatally amygdala-lesioned male. The replacement male had been reared alone with his mother for 10 months. Following weaning, he was housed with an age-matched female infant until being introduced to his current cohort at approximately 1 year and 3 months of age. The replacement subject was accepted by the social group and had lived with the cohort for approximately 4 months prior to testing.

\section{Surgical Procedures}

The surgical procedures are summarized below and have been described in detail in previous publications (Bauman et al., 2004a, 2004b). On the day of surgery, the infants were initially anesthetized with ketamine hydrochloride $(15 \mathrm{mg} / \mathrm{kg} \mathrm{im})$ and medetomidine $(30 \mu \mathrm{g} / \mathrm{kg})$ and were then placed in an MRI-compatible stereotaxic apparatus (Crist Instruments, Damascus, MD). The infant's brain was imaged with a General Electric 1.5 T Gyroscan magnet; 1.0-mm-thick sections were taken with a T1-weighted inversion recovery pulse sequence (return time $[\mathrm{TR}]=21 \mathrm{~ms}$, echo time $[\mathrm{TE}]=7.9 \mathrm{~ms}$, number of excitations $[\mathrm{NEX}]=3$, field of view $[\mathrm{FOV}]=$ $8 \mathrm{~cm}$, matrix $=256 \times 256$ ). From these images, we determined the location of the amygdala or hippocampus and calculated the coordinates for the ibotenic acid injections. Infants were ventilated, and vital signs were monitored throughout the surgery. A stable level of anesthesia was maintained by using a combination of isoflurane $(\approx 1.0 \%$, varied as needed to maintain an adequate level of anesthesia) and intravenous infusion of fentanyl (7-10 $\mu \mathrm{g} / \mathrm{kg} / \mathrm{hr})$. Following a midline incision, the skin was laterally displaced to expose the skull; two craniotomies were made over the amygdala or the hippocampus, depending on the predetermined lesion condition; and the dura was reflected to expose the surface of the brain. Ibotenic acid (Biosearch Technologies, Novato, CA; $10 \mathrm{mg} / \mathrm{ml}$ in $0.1 \mathrm{M}$ phosphate buffered saline) was injected simultaneously bilaterally into the 
amygdala or hippocampus with 10- $\mu 1$ Hamilton syringes (26-gauge beveled needles) at a rate of $0.2 \mu \mathrm{l}$ per minute. Sham-operated controls underwent the same presurgical preparations and received a midline incision, and the skull was exposed. The control animals were maintained under anesthesia for the average duration of the lesion surgeries, and the fascia and skin were sutured in two separate layers. Following the surgical procedure, all infants were monitored by a veterinarian and returned to their mothers once they were fully alert.

\section{Lesion Analysis}

T2-weighted MRIs were obtained 10 days after surgery so that we could examine the extent of edema associated with the lesion. We evaluated the hyperintense T2-weighted signal for each of the 16 lesion subjects to confirm the general target and extent of the lesions (i.e., amygdala lesion sparing the hippocampus or hippocampus lesion sparing the amygdala). Their brains were imaged with a General Electric 1.5 T Gyroscan magnet; 1.5 -mm thick sections were taken with a $\mathrm{T} 2$-weighted inversion recovery pulse sequence $(\mathrm{TR}=4,000 \mathrm{~ms}, \mathrm{TE}=102 \mathrm{~ms}, \mathrm{NEX}=3, \mathrm{FOV}=8 \mathrm{~cm}$, matrix $=256 \times 256$ ). Additional lesion confirmation was provided by T1-weighted MRIs obtained at approximately 4 years of age. The monkeys' brains were scanned with a General Electric 1.5 T Signa MRI system; 1-mm-thick sections were taken with a T1-weighted three-dimensional axial-spoiled gradient sequence $(\mathrm{TR}=22.0 \mathrm{~ms}, \mathrm{TE}=7.9 \mathrm{~ms}, \mathrm{NEX}=3$, $\mathrm{FOV}=16 \mathrm{~cm}$, matrix $=256 \times 256$ ).

\section{Behavioral Observations and Statistical Analysis}

Behavioral data were collected with The Observer software (Noldus, Sterling, VA; Noldus, 1991) by trained observers demonstrating an interobserver reliability greater than $90 \%$ (agreements/[agreements + disagreements] $\times 100$ ). Analyses of variance followed by Fisher's protected least significant difference post hoc tests (with a significance level of $p<.05$ ) were used for data analyses. Because of heterogeneous variation, all latency data were transformed with the $\ln (x+1)$ transformation to conform to normal distribution requirements.

\section{Evaluation of Pretest Food Motivation}

Although each subject had been given marshmallows as enrichment throughout its life, a formal test of the willingness and motivation to retrieve miniature marshmallows from an affixed novel container was essential in confirming that marshmallows were indeed a preferred food item. Therefore, 1 week prior to the dominance test, each subject's willingness to retrieve marshmallows from the food container was evaluated. The marshmallows were placed in a plastic food container $(15 \mathrm{~cm}$ long $\times$ $13 \mathrm{~cm}$ wide $\times 8 \mathrm{~cm}$ high) with a 4-cm circular opening located at the top. Willingness to retrieve the marshmallows (as indicated by the frequency of reaches into the container) was assessed in two different contexts. The first assessed the ability of each subject to retrieve marshmallows from the food container when removed from its social group. Subjects were temporarily relocated to individual holding cages $(61 \mathrm{~cm}$ long $\times 66 \mathrm{~cm}$ wide $\times 81 \mathrm{~cm}$ high) within the same room and given free access to the container filled with marshmallows during a single 15 -min session. The second context evaluated each subject's ability to retrieve marshmallows with all members of the cohort present (social context). To minimize competition between subjects prior to the formal test of dominance, we attached six identical containers filled with marshmallows to the inside of each cohort's large home enclosure $(8 \mathrm{~cm}$ apart, with the bottom approximately $25 \mathrm{~cm}$ above the floor). The adult male and female were first removed from the home enclosure, and the 6 juvenile subjects were relocated to wire-mesh holding chutes attached to the back of their home enclosure. A technician then entered the cage and strapped the six baited containers to the front of the cage. Once the containers were securely fastened, the first technician exited the cage, and a second technician released all 6 subjects simultaneously into the cage. Once released, the subjects were allowed access to the six containers for $15 \mathrm{~min}$. There were no changes in normal diet or feeding schedules associated with these tests.

\section{Dominance Test Procedure}

Each cohort consisting of 2 amygdala-lesioned, 2 hippocampuslesioned, and 2 sham-operated juvenile subjects was tested in their home enclosure $(2.13$-m width $\times 3.35$-m diameter $\times 2.44$-m height $)$. Dominance testing comprised two distinct phases, with a 5-month interval separating Phase 1 from Phase 2. During each phase, we assessed dominance by allowing each cohort free access to a single container filled with marshmallows for a $15-\mathrm{min}$ session between $1 \mathrm{p} . \mathrm{m}$. and $2 \mathrm{p} . \mathrm{m}$. This procedure was repeated five additional times over a 2 -week period, resulting in six 15-min sessions per cohort per testing phase.

On the day of the dominance test, the adult male and female were first removed from the testing area, and the 6 juvenile subjects of a given cohort were relocated to wire-mesh holding chutes attached to the back of their home enclosure. A technician then entered the cage and strapped the baited container to the front of the cage $(25 \mathrm{~cm}$ above the floor). Once the container was securely fastened in the cage and the technician exited, a second technician released all 6 subjects simultaneously into the cage. Subjects were allowed free access to the container for $15 \mathrm{~min}$ (see Figure 1). At the conclusion of the test, the container was removed and the adults were returned to the social group.

\section{Dominance Test Data Collection}

Access to food measures. We quantified access to the preferred food (marshmallows) by recording each subject's latency to first approach within arm's reach of the container and each subject's latency to reach its hand into the container, as well as the order (ranging from $1=$ reached first to $6=$ reached last ) in which each of the 6 subjects of a given cohort first reached into the container. For those instances in which a subject never reached during a single test session, the maximum value of 6 was assigned. Reach orders were then summed for each subject across each test session (6 sessions per phase, 12 sessions total). As was done with reach order, if a subject never approached or reached during the 15-min test session, the maximum value of $900 \mathrm{~s}$ was assigned. An overall frequency of reaching was also obtained by recording the number of times each subject was observed inserting its hand into the container throughout the 15-min session. In addition, the frequency and duration of time spent in proximity to the container (within arm's reach) was also recorded (see Table 1).

Access to food in the first minute of testing. Preferential access to food is an indication of social dominance (Belzung \& Anderson, 1986). Given that the most dominant members of a group will generally gain initial access to a restricted food resource, we conducted a separate analysis of the food access measures described above limited to the first minute of dominance testing.

Behavioral measures. All frequencies of species-typical agonistic behaviors, such as aggression, displacements, fear grimaces, and screams, were recorded with the identity of the initiator and recipient of each agonistic interaction noted (Table 1). A linear dominance hierarchy was constructed for each cohort on the basis of the directionality of recorded dyadic aggressive interactions between subjects (Bastian et al., 2003; Higley, Mehlman, et al., 1996). Linear rank indices (1-6) were derived by using the formula $x /(n-1)$, with $x$ signifying the total number of monkeys each subject defeated and $n-1$ representing the number of monkeys each subject could possibly defeat (maximum of 5 within their respective cohorts). A subject was considered more dominant than another subject when it initiated more aggressive behaviors than it received during interactions with that subject. 


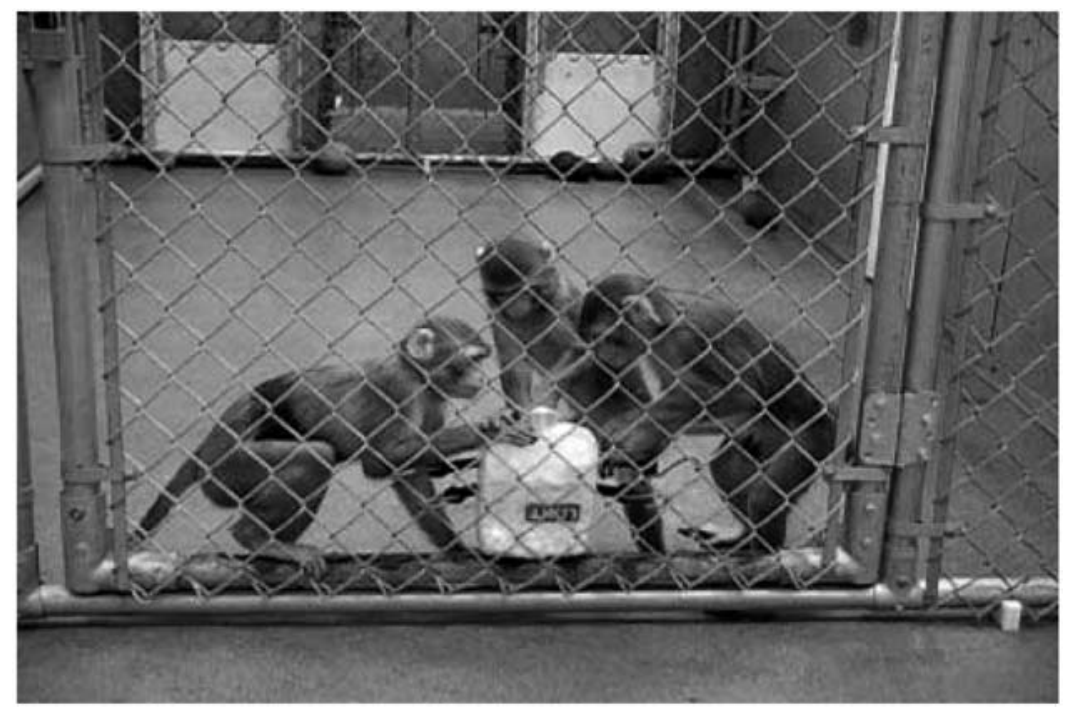

Figure 1. Photograph illustrating the testing enclosure in which a container was placed that allowed limited access to a preferred food (marshmallows).

\section{Social Group Observations}

In addition to the formal assessment of dominance, we conducted weekly social group observations within each cohort's home enclosure to provide information on dominance-related behaviors under normative conditions (i.e., in the absence of a preferred resource). Each subject was observed on 40 separate occasions during 5-min focal samples. We observed subjects in a predetermined pseudorandom order by using a previously described catalog of age-appropriate, species-typical behaviors (Bauman et al., 2004b).

\section{Results}

\section{MRI and Histological Evaluation of Lesions}

Analysis of T2-weighted coronal images obtained 10 days following the surgery indicated that the ibotenic acid injections were focused in the amygdala or hippocampus as planned. This is based on observations of the location of hyperintense signals attributed to transient brain edema at the sight of the injection. Coronal images through the midportion of the amygdala for all cases were illustrated in previous publications (Bauman et al., 2004a, 2004b). The extent of the targeted lesion was confirmed in 1 amygdala-lesioned subject that died because of an unrelated illness. Histological evaluation of the brain in this case confirmed that neurons throughout much of the amygdala were damaged bilaterally and that extraneous damage to surrounding structures was minimal and generally not bilaterally symmetrical (see Figure 2 in Bauman et al., 2004b).

Analysis of a second series of structural MRIs performed when the subjects were approximately 4 years of age provided additional confirmation of the lesions. All 8 amygdala-lesioned subjects demonstrated substantial bilateral damage to the amygdaloid complex, as indicated by clear shrinkage of the amygdala and/or expansion of the ventricles into space formerly occupied by the amygdala (see Figure 2). If there was any sparing of amygdala

Table 1

Dominance Test Ethogram

\begin{tabular}{|c|c|}
\hline Behavior & Description \\
\hline \multicolumn{2}{|r|}{ Duration behavior } \\
\hline Proximity to food container & Within arm's reach of the food container for more than $3 \mathrm{~s}$ \\
\hline \multicolumn{2}{|r|}{ Frequency behavior } \\
\hline Contact aggression & Grab, hit, bite, or slap \\
\hline Approach (to food container) & Directed movement within arm's reach of container \\
\hline Avoid & Withdraw from the container due to the arrival of another subject \\
\hline Displacement & When another subject approaches and "takes the place" of the other subject \\
\hline Fear grimace & Upper and lower lips retracted, exposing teeth \\
\hline Flee & Rapid movement away from another subject \\
\hline Reach & Hand or arm inserted into food container \\
\hline Scream & High-pitched, high-intensity vocalization \\
\hline Threat & One or more of the following: open-mouth stare, head bob, lunge \\
\hline
\end{tabular}


tissue, it was limited to the most caudal aspects of the amygdala, perhaps including the central nucleus. Unintended damage to cortex lying adjacent to the amygdala appeared to be minimal. Damage to the hippocampus in the amygdala-lesioned subjects was limited to its rostral portion. Analysis of the hippocampus lesions revealed nearly complete bilateral damage for all cases, with minimal sparing of the extreme rostral and caudal portions (Figure 2 ). Unintended damage to the amygdala was not observed in any of the 8 hippocampus-lesion cases, and only slight damage was found in the surrounding parahippocampal cortex in one of the cases. Lesion parameters were designed to minimize inadvertent damage to the amygdala or surrounding cortices.

\section{Pretest Food Motivation}

Subjects did not differ in the frequency of reaches into the container when tested alone, $F(2,21)=0.173, p=.8425$, and when tested in a group with access to multiple containers, $F(2$, $21)=0.846, p=.4431$. Thus, lesion condition did not affect the subject's motivation to obtain the limited preferred resource (marshmallows).

\section{Dominance Test Results}

\section{Overview}

Although dominance testing consisted of two distinct phases of data collection so that we could evaluate the stability of the hierarchies over a 6-month period, there were not many significant differences found between Phase 1 and Phase 2; thus, data were summed across the two phases. Several indices of social dominance were measured, including food access, frequency of agonistic behaviors (Table 1), and the calculation of a linear dominance hierarchy.

\section{Access to Food}

Order of food access. Experimental groups demonstrated significant differences on the measures of initial access to food, with the exception of approaches to the container. Lesion effects were found for the order of reaching, $F(2,21)=19.877, p<.0001$; control subjects reached before amygdala- and hippocampuslesioned subjects ( $p<.0001$ and $p=.0023$, respectively), and hippocampus-lesioned subjects reached before amygdala-lesioned subjects ( $p=.0103$; see Figure 3 ). Lesion effects were also found for the latency to first approach the container, $F(2,21)=9.254$, $p=.0013$, and to first reach into the container, $F(2,21)=6.450$, $p=.0066$. Amygdala-lesioned subjects took longer to approach the container than did control and hippocampus-lesioned subjects ( $p=.0004$ and $p=.0094$, respectively), and they also took longer to reach into the container than did control and hippocampuslesioned subjects ( $p=.0004$ and $p=.0094$, respectively).

Access to food in the first minute of testing. Experimental groups differed markedly in their access to the food container during the first minute of testing when competition was presumably greatest (see Table 2). The total number of reaches into the food container, for example, during the first minute of testing was significantly different across experimental groups, with control subjects reaching more frequently into the container than both amygdala- and hippocampus-lesioned subjects, $F(2,21)=12.085$, $p=.0003 ; p<.0001$ and $p=.0100$, respectively. Lesion effects were also found for the number of times subjects were observed in proximity to the container during the first minute of testing. Control and hippocampus-lesioned subjects were more frequently in proximity to the container than the amygdala-lesioned subjects, $F(2,21)=5.343, p=.0133 ; p=.0044$ and $p=.0377$, respectively. Lesion effects were also found for duration of proximity, with control subjects spending more time near the food container than both amygdala- and hippocampus-lesioned subjects, $F(2$, $21)=6.039, p=.0085 ; p=.0025$ and $p=.0419$, respectively. It is interesting to note that the frequency of approaches to the container (within arm's reach) during the first minute of testing did not differ across the experimental groups, $F(2,21)=2.149, p=$ .1416 , indicating that all subjects were initially motivated to approach the food area.

Overall access to food. By the second minute of testing the difference in reaching between the experimental groups was not significant, $F(2,21)=0.192, p=.8271$, suggesting that maximum efforts to monopolize the food container took place for only a short time. Indeed, lesion groups did not differ on any food access measures across the entire 15-min dominance test.

\section{Frequency of Dominance-Related Behaviors}

Lesion effects were consistently found for the production of behaviors associated with aggression (see Table 3). Amygdalalesioned subjects demonstrated fewer instances of contact aggression (i.e., hits, bites, slaps) than did control subjects, $F(2,21)=$ $3.570 ; p=.0463$ and $p=.0143$, respectively; and they displaced subjects less frequently than did both control and hippocampuslesioned subjects, $F(2,21)=5.361, p=.0132 ; p=.0059$ and $p=$ .0191 , respectively. Taking together all behaviors associated with the initiation of aggression (i.e., threats, displacements, and contact aggression), amygdala-lesioned subjects initiated fewer aggressive behaviors than did both control and hippocampus-lesioned subjects, $F(2,21)=7.007, p=.0047 ; p=.0014$ and $p=.0234$, respectively (see Figure 4). No lesion effects were found for which group received the aggressive behaviors. This suggests that the lack of aggression exhibited by the amygdala subjects did not make them preferential targets of aggression. Amygdala-lesioned subjects were, however, avoided less than either the hippocampuslesioned or control subjects as other subjects approached the food container, $F(2,21)=3.920, p=.0358 ; p=.0185$ and $p=.0338$, respectively. This was perhaps an indication that the other subjects anticipated less aggression from the amygdala-lesioned animals.

Amygdala-lesioned subjects also differed from control and hippocampus-lesioned subjects in their use of species-typical fear behaviors. Amygdala-lesioned subjects produced more screams than did either control or hippocampus-lesioned subjects, $F(2$, $21)=3.641, p=.0439 ; p=.0205$ and $p=.0459$, respectively. Although the production of fear grimaces or flees failed to reach significance individually, when all fear behaviors (i.e., fear grimaces, flees, and screams) were grouped and analyzed together, amygdala-lesioned subjects produced more fear behaviors than did either control or hippocampus-lesioned subjects, $F(2,21)=4.592$, $p=.0222 ; p=.0078$ and $p=.0478$, respectively (see Figure 5). It is interesting that there were more fear behaviors directed at the control animals than either the hippocampus- or amygdala- 

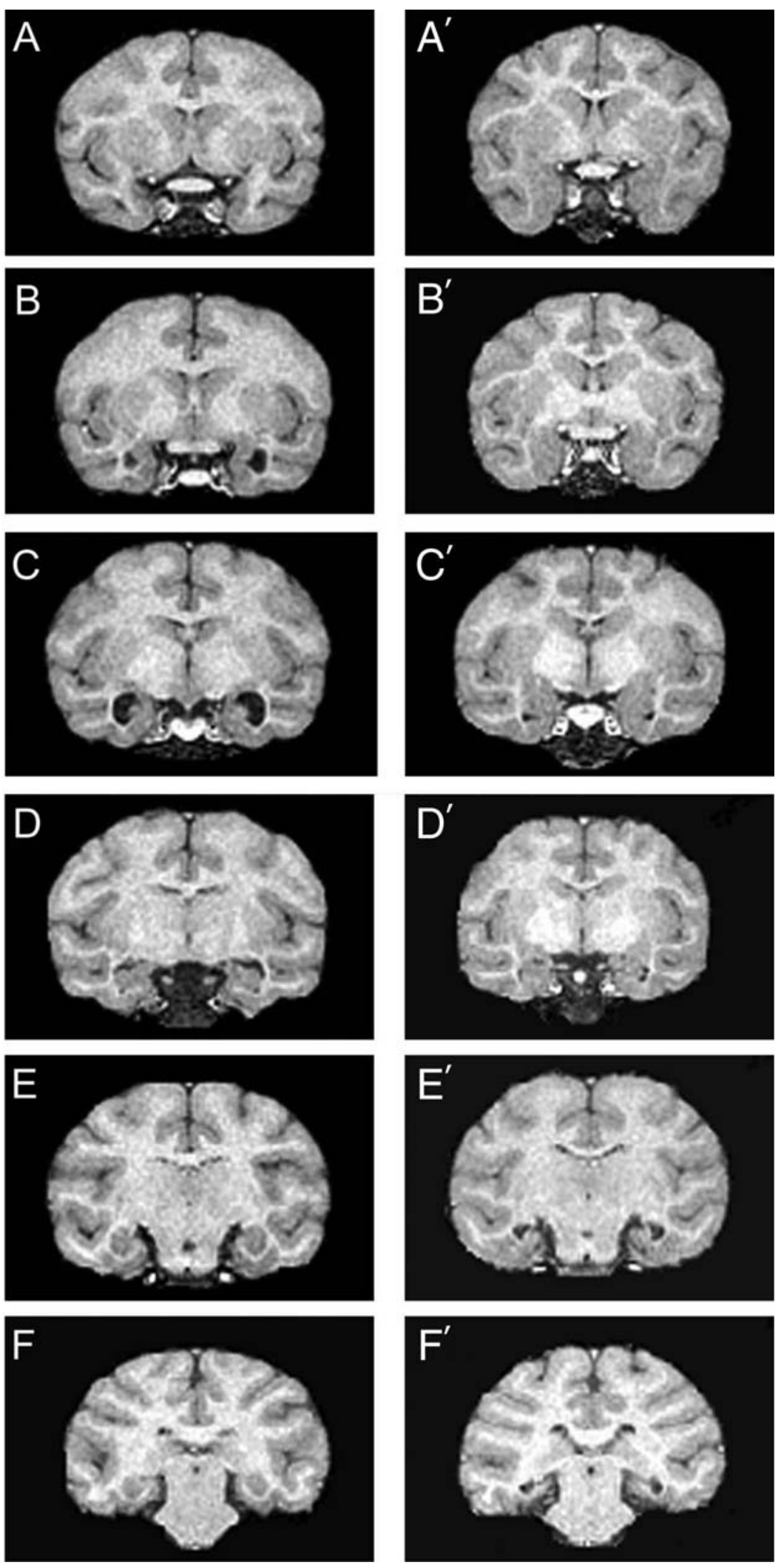
lesioned subjects, $F(2,21)=8.470, p=.0020 ; p=.0006$ and $p=$ .0128 , respectively (Table 3 ).

\section{Linear Dominance Rank}

Amygdala-lesioned subjects consistently ranked lower than both control and hippocampus-lesioned subjects, $F(2,21)=11.605$, $p=.0004 ; p=.0001$ and $p=.0071$, respectively (see Figure 6). There were no changes in social rank for any of the four cohorts between Phases 1 and 2 of testing.

\section{Social Group Interactions}

Subjects were observed weekly in their social groups to provide supplemental data on baseline propensities for aggressive and fearful behaviors. Amygdala-lesioned subjects initiated less contact aggression than did control and hippocampus-lesioned subjects, $F(2,21)=3.737, p=.0409 ; p=.0200$ and $p=.0405$, respectively. Although threats were also scored during social group observations, the vast majority of these behaviors were playful threats (i.e., threats occurring during the context of play, a presumably benign context) as opposed to aggressive threats (i.e., threats occurring during aggressive encounters). Given the subjective nature of these behaviors, threats were not included in the analysis of aggressive behaviors. The frequency of displacements was not statistically significant, $F(2,21)=3.304, p=.0565$, although the effect of lesion was significant when both contact aggression and displacements were grouped. Amygdala-lesioned subjects again initiated less aggression and displacements than did control and hippocampus-lesioned subjects, $F(2,21)=4.717, p=$ $.0203 ; p=.0092$ and $p=.0264$, respectively. Unlike the results for the formal test of dominance, there were no consistent lesion effects for the expression of fear behaviors (i.e., fear grimace, scream, and flee) during social group observations. However, when interactions involving the adult male and female of each cohort were excluded, the effect of lesion on fear grimaces was significant, $F(2,21)=5.034, p=.0164$. Amygdala-lesioned subjects generated more fear grimaces toward other juvenile members of their respective cohorts than did either control or hippocampus-lesioned subjects $(p=.0079$ and $p=.0201$, respectively).

\section{Discussion}

\section{Amygdala Damage Affects Social Rank}

In the present study, control and hippocampus-lesioned subjects ranked higher than amygdala-lesioned subjects on behavioral indices of social dominance during limited access to a preferred food source. All subjects, irrespective of lesion condition, were moti-

\section{Reach Order}

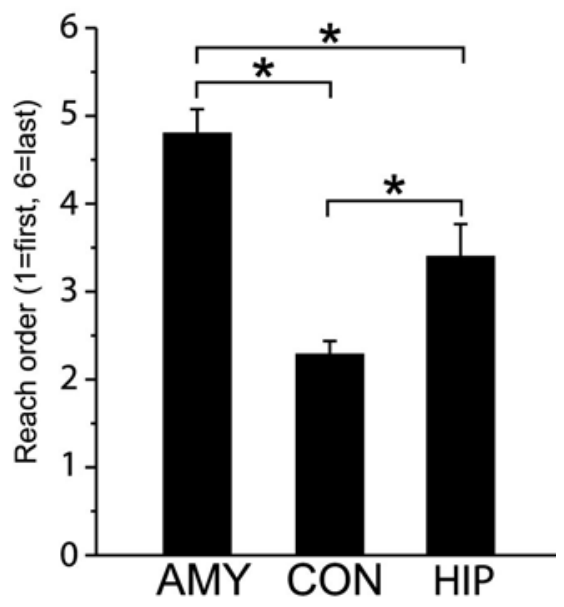

Figure 3. Graph illustrating mean order of reaching into the marshmallow container $(1=$ first subject in a group, $6=$ last subject in a group $)$ during dominance testing. Each bar represents the average order of first reaching into the container ( \pm SEM per 15 -min observation period). Asterisks denote significant post hoc Fisher's protected least significant difference tests $(p<.05)$. AMY $=$ amygdala-lesioned subjects; $\mathrm{CON}=$ sham-operated controls; HIP $=$ hippocampus-lesioned subjects.

vated to retrieve the food when tested individually or when multiple food containers were available. Moreover, lesion groups did not differ in food access across the entire 15-min span of the dominance test, indicating that all subjects were motivated to obtain the food reward and were eventually capable of doing so. There were, however, pronounced differences among lesion groups in gaining access to the preferred food during the first minute of testing, when competition was presumably greatest. In general, amygdala-lesioned subjects were the last subjects to gain access to the food, an indication of low social rank (Belzung \& Anderson, 1986). During these studies, we induced competition among group members as an assay of social dominance. However, dominance relationships are based on the collective pattern of previous interactions between two individuals (Bernstein, 1981). Given that these subjects had been raised together since the first month of life, it is likely that the amygdala-lesioned subjects' limited access to food during the dominance task reflects on overall diminished capacity to obtain high rank within their social group.

\section{Dominance in Macaque Monkeys}

To address the underlying cause of the low social rank observed in the amygdala-lesioned subjects, it is first necessary to consider

Figure 2 (opposite). T1-weighted coronal MRIs of a brain with a representative amygdala lesion (A-F, left panels) and a second brain with a representative hippocampus lesion $\left(\mathrm{A}^{\prime}-\mathrm{F}^{\prime}\right.$, right panels). Panels $\mathrm{A}-\mathrm{C}$ illustrate complete bilateral amygdala damage from 1 of the amygdala-lesioned subjects. Panels $\mathrm{A}^{\prime}-\mathrm{C}^{\prime}$, which are located at approximately the same rostrocaudal levels as $\mathrm{A}-\mathrm{C}$, illustrate sparing of the amygdala in 1 of the hippocampus-lesioned subjects. Panels $\mathrm{D}^{\prime}-\mathrm{F}^{\prime}$ illustrate complete bilateral hippocampus damage from 1 of the hippocampus-lesioned subjects. Panels D-F illustrate sparing of the hippocampus in 1 of the amygdala-lesioned subjects. 


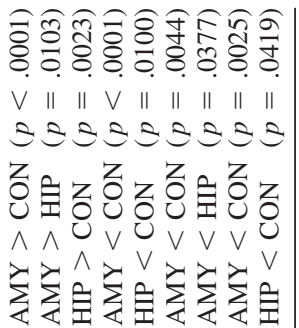

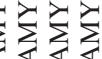

what behavioral attributes are typically required to obtain and maintain dominance status. In free-ranging rhesus monkeys, prediction of social rank is closely linked to the dominance status of kin, with high-ranking mothers producing high-ranking offspring (Missakian, 1972; Sade, 1967). When such naturalistic determinants of dominance are absent from group composition (as was the case following weaning at 6 months of age in the present study), social rank is likely influenced by a variety of other factors, including an individual's temperament, physical attributes, and ability to form coalitions with other group members (Bernstein \& Ehardt, 1985; Bernstein \& Mason, 1963; Higley, King, et al., 1996).

A recent model of social dominance suggests that primates are striving to have their own way whenever possible (i.e., to move freely within the group, to interact with whomever they choose, and to have unimpeded access to desirable resources; Mason, 1993). In situations where the interests of one individual impinge on the well-being of another individual, conflict is likely to arise, often accompanied by aggression. Dominance relationships are determined by the outcomes of such agonistic social interactions (Sade, 1967). Though not all agonistic encounters result in physical aggression, milder forms of aggression, such as threats, play a critical role in periodically reinforcing dominance relationships (Bernstein \& Ehardt, 1985). Indeed, the most aggressive primate species demonstrate the most strictly defined and enforced dominance hierarchies (Thierry, 1985). It is important to note, however, that aggression alone does not determine social rank. In highly social animals, such as rhesus macaques, social status relies heavily on the ability to recruit allies and form coalitions with fellow group members (Thierry, 1990). Thus, high-ranking individuals must display an appropriate combination of both agonistic (i.e., aggressive and submissive) and affiliative social signals in order to recruit allies and consistently elicit submissive responses from lower ranking group members (de Waal, 1993).

\section{Potential Causes of Low Social Rank}

To gain insight into the underlying cause of low rank in the amygdala-lesioned subjects, we also evaluated the use of speciestypical social signals associated with dominance status, focusing our observations on fearful and aggressive behaviors. During the dominance task, the amygdala-lesioned subjects consistently initiated fewer aggressive behaviors and displayed more fear behaviors than did either control or hippocampus-lesioned subjects. On the basis of the requirements of attaining high social rank outlined above, it is possible that this abnormal pattern of fear and aggression may underlie the low social rank of the amygdala-lesioned subjects.

If the amygdala-lesioned subjects experience a continuously heightened sense of fear of conspecifics, this would presumably impact their ability to engage in social conflicts. Although the amygdala-lesioned subjects might desire a food resource, their fear of other monkeys may prevent them from directly competing with conspecifics to obtain the food. Abnormal social fear may also prevent the amygdala-lesioned subjects from engaging in social interactions required to recruit and maintain allies and to form coalitions. Given that dominance relationships are defined by a 
Table 3

Dominance Task Behavioral Measures

Task totals (Phases $1 \& 2)$

\begin{tabular}{|c|c|c|c|c|c|}
\hline Behavioral measure & $\begin{array}{l}\text { AMY } \\
\text { frequency }\end{array}$ & $\begin{array}{l}\mathrm{CON} \\
\text { frequency }\end{array}$ & $\begin{array}{c}\text { HIP } \\
\text { frequency }\end{array}$ & Lesion effect & Post hoc \\
\hline $\begin{array}{l}\text { Contact aggression } \\
\text { initiate }\end{array}$ & $0.417 \pm 0.291$ & $2.333 \pm 0.648$ & $1.313 \pm 0.518$ & $F(2,21)=3.570, p=.0463$ & $\mathrm{AMY}<\mathrm{CON}(p=.0143)$ \\
\hline $\begin{array}{l}\text { Contact aggression } \\
\text { receive }\end{array}$ & $1.656 \pm 0.405$ & $0.906 \pm 0.338$ & $1.500 \pm 0.386$ & $F(2,21)=1.100, p=.3513$ & \\
\hline Displace initiate & $0.052 \pm 0.027$ & $2.135 \pm 0.600$ & $1.781 \pm 0.579$ & $F(2,21)=5.361, p=.0132$ & $\begin{array}{l}\mathrm{AMY}<\mathrm{CON} \quad(p=.0059) \\
\mathrm{AMY}<\mathrm{HIP} \quad(p=.0191)\end{array}$ \\
\hline Displace receive & $1.813 \pm 0.492$ & $0.979 \pm 0.305$ & $1.146 \pm 0.273$ & $F(2,21)=1.423, p=.2634$ & \\
\hline Threat initiate & $0.354 \pm 0.175$ & $1.510 \pm 0.389$ & $1.156 \pm 0.379$ & $F(2,21)=3.232, p=.0598$ & \\
\hline Threat receive & $1.000 \pm 0.253$ & $0.771 \pm 0.258$ & $1.260 \pm 0.257$ & $F(2,21)=0.916, p=.4154$ & \\
\hline Avoid initiate & $0.979 \pm 0.289$ & $0.760 \pm 0.326$ & $1.208 \pm 0.326$ & $F(2,21)=0.508, p=.6091$ & \\
\hline Avoid receive & $0.271 \pm 0.224$ & $1.396 \pm 0.285$ & $1.271 \pm 0.354$ & $F(2,21)=3.920, p=.0358$ & $\begin{array}{l}\text { AMY }<\text { CON } \quad(p=.0185) \\
\text { AMY }<\text { HIP } \quad(p=.0338)\end{array}$ \\
\hline $\begin{array}{l}\text { Fear grimace } \\
\text { initiate } \\
\text { Fear grimace }\end{array}$ & $1.938 \pm 1.091$ & $0.052 \pm 0.031$ & $0.344 \pm 0.117$ & $F(2,21)=2.566, p=.1007$ & \\
\hline receive & $0.073 \pm 0.053$ & $1.615 \pm 0.636$ & $0.312 \pm 0.185$ & $F(2,21)=4.677, p=.0209$ & $\begin{array}{l}\mathrm{AMY}<\mathrm{CON} \quad(p=.0098) \\
\mathrm{HIP}<\mathrm{CON} \quad(p=.0257)\end{array}$ \\
\hline Flee initiate & $1.437 \pm 0.443$ & $0.792 \pm 0.363$ & $1.510 \pm 0.313$ & $F(2,21)=1.103, p=.3503$ & \\
\hline Flee receive & $0.344 \pm 0.235$ & $2.271 \pm 0.447$ & $0.948 \pm 0.340$ & $F(2,21)=7.865, p=.0028$ & $\begin{array}{ll}\mathrm{AMY}<\mathrm{CON} & (p=.0009) \\
\mathrm{HIP}<\mathrm{CON} & (p=.0146)\end{array}$ \\
\hline Scream initiate & $2.479 \pm 1.030$ & $0.302 \pm 0.168$ & $0.635 \pm 0.208$ & $F(2,21)=3.641, p=.0439$ & $\begin{array}{l}\mathrm{AMY}>\mathrm{CON} \quad(p=.0205) \\
\mathrm{AMY}>\mathrm{HIP} \quad(p=.0459)\end{array}$ \\
\hline $\begin{array}{l}\text { Scream receive } \\
\text { All aggressive }\end{array}$ & $0.187 \pm 0.111$ & $0.906 \pm 0.198$ & $0.708 \pm 0.244$ & $F(2,21)=3.728, p=.0411$ & $\mathrm{AMY}<\mathrm{CON}(p=.0152)$ \\
\hline behaviors initiate & $0.823 \pm 0.467$ & $5.979 \pm 1.095$ & $4.250 \pm 1.237$ & $F(2,21)=7.007, p=.0047$ & $\begin{array}{l}\text { AMY }<\mathrm{CON} \quad(p=.0014) \\
\mathrm{AMY}<\mathrm{HIP} \quad(p=.0234)\end{array}$ \\
\hline $\begin{array}{l}\text { All aggressive } \\
\text { behaviors receive } \\
\text { All fear behaviors }\end{array}$ & $4.469 \pm 0.603$ & $2.656 \pm 0.825$ & $3.906 \pm 0.692$ & $F(2,21)=1.694, p=.2080$ & \\
\hline initiate & $5.854 \pm 1.809$ & $1.146 \pm 0.468$ & $2.490 \pm 0.592$ & $F(2,21)=4.592, p=.0222$ & $\begin{array}{l}\mathrm{AMY}>\mathrm{CON} \quad(p=.0078) \\
\mathrm{AMY}>\operatorname{HIP} \quad(p=.0478)\end{array}$ \\
\hline $\begin{array}{l}\text { All fear behaviors } \\
\text { receive }\end{array}$ & $0.604 \pm 0.334$ & $4.792 \pm 1.073$ & $1.969 \pm 0.593$ & $F(2,21)=8.470, p=.0020$ & $\begin{array}{l}\mathrm{AMY}<\mathrm{CON} \quad(p=.0006) \\
\mathrm{HIP}<\mathrm{CON} \quad(p=.0128)\end{array}$ \\
\hline
\end{tabular}

Note. Frequency for all behavioral measures was quantified during dominance testing. Average number of occurrences (frequency) $\pm S E M$ per testing session is shown for amygdala-lesioned subjects (AMY), sham-operated controls (CON), and hippocampus-lesioned subjects (HIP).

cumulative history of agonistic encounters, one would expect that the amygdala-lesioned subjects demonstrated a similar behavioral profile (i.e., low rank and heightened fear behaviors) in the absence of the artificially induced food competition. Indeed, our observations of the amygdala-lesioned subjects in their juvenile social groups revealed a similar trend of heightened fearful or submissive behaviors directed to other juvenile subjects. Moreover, this population of amygdala-lesioned subjects demonstrated an abnormal and pervasive fear of unthreatening conspecifics during the first year of life, which may have causally contributed to their low social rank (Bauman et al., 2004a, 2004b). Though species-typical fear responses are an appropriate means of conveying subordination (Hinde \& Spencer-Booth, 1967), it is possible that the excessive fear produced by the amygdala-lesioned subjects may have resulted in a diminished capacity to compete, form coalitions, and subsequently obtain high social status earlier in development.

In contrast to heightened fear responses, the amygdala-lesioned subjects demonstrated a diminished capacity to use species-typical aggressive behaviors. Though the amygdala-lesioned subjects de- scribed in the present study are capable of producing aggressive signals, they used these behaviors less frequently than did control and hippocampus-lesioned subjects. As predicted by the model of social dominance described above, the control and hippocampuslesioned subjects responded to a provocative stimulus (the limited food reward) with species-typical social conflict and aggression, resulting in preferential access to the food. In contrast, the amygdala-lesioned subjects rarely used species-typical aggressive behaviors and therefore gained access to the food after the control and hippocampus-lesioned subjects. It is interesting to note that the amygdala-lesioned subjects also initiated fewer aggressive behaviors when observed in their weekly social groups (in the absence of the competitive food task). An inability to respond to social conflict with species-typical aggression would thus prevent the amygdala-lesioned subjects from ever obtaining high social rank within their group. Indeed, diminished use of aggression was first observed in the amygdala-lesioned subjects at 12 months of age (Bauman et al., 2004b). This pattern of behavior appears to have persisted and likely contributed to the low rank of the amygdalalesioned subjects. 


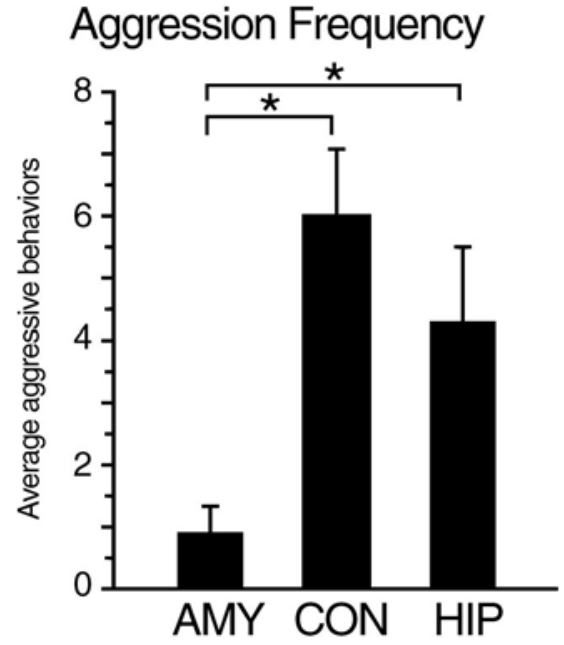

Figure 4. Graph illustrating mean frequency of aggressive behaviors (i.e., threats, displacements, and contact aggression) produced during dominance testing ( $\pm S E M$ per 15 -min observation period). Asterisks denote significant post hoc Fisher's protected least significant difference tests $(p<.05)$. AMY $=$ amygdala-lesioned subjects; $\mathrm{CON}=$ sham-operated controls; HIP $=$ hippocampus-lesioned subjects.

\section{Previous Lesion Studies}

Lesions of the amygdala have long been associated with deficits in fear processing in nonhuman primates (Izquierdo, Suda, \& Murray, 2005; Kalin, Shelton, Davidson, \& Kelley, 2001; Weiskrantz, 1956; Zola-Morgan, Squire, Alvarez-Royo, \& Clower, 1991) and with decreases in aggression and increases in tameness in a variety of species (reviewed in Kling, 1992). Given that appropriate regulation of both fear and aggression are essential for

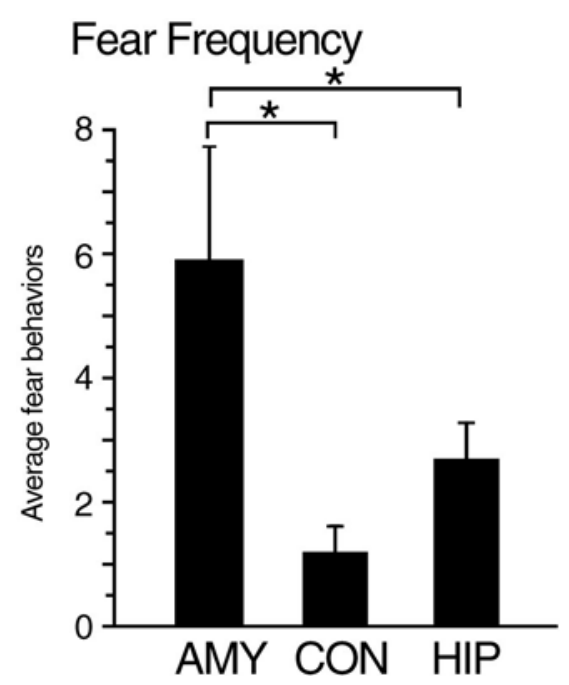

Figure 5. Graph illustrating mean frequency of fear behaviors (i.e., fear grimaces, flees, and screams) produced during dominance testing ( \pm SEM per 15-min observation period). Asterisks denote significant post hoc Fisher's protected least significant difference tests $(p<.05)$. AMY $=$ amygdala-lesioned subjects; $\mathrm{CON}=$ sham-operated controls; HIP = hippocampus-lesioned subjects.

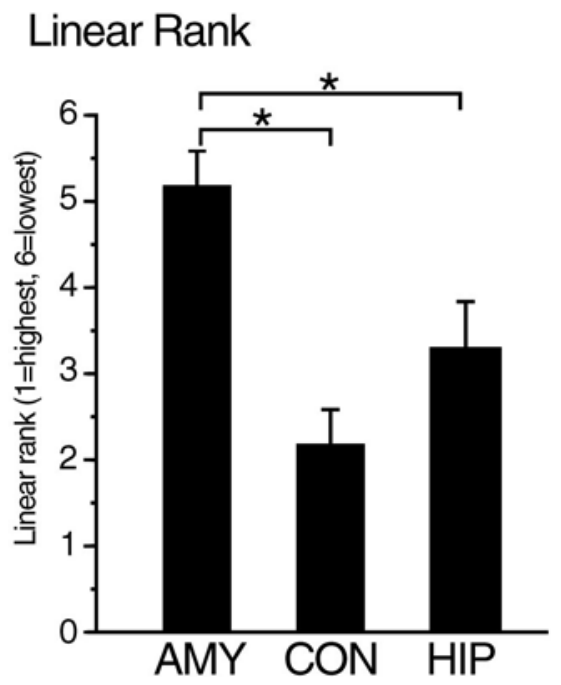

Figure 6. Graph illustrating mean linear hierarchical rank $(1=$ highest rank, $6=$ lowest rank) of subjects during dominance testing ( $\pm S E M$ per 15-min observation period). Asterisks denote significant post hoc Fisher's protected least significant difference tests $(p<.05)$. AMY $=$ amygdalalesioned subjects; $\mathrm{CON}=$ sham-operated controls; HIP $=$ hippocampuslesioned subjects.

obtaining and maintaining high social rank (Sade, 1967), it is not surprising that damage to the amygdala profoundly affects social dominance. Previous amygdala lesion studies have suggested that the combination of heightened social fear and diminished aggressive capacities may be particularly detrimental in the attainment of social dominance (Kling, 1972). Our results are consistent with previous studies in which amygdala damage followed by reintroduction to adult primate social groups was associated with diminished social dominance (Kling \& Cornell, 1971; Plotnik, 1968; Rosvold et al., 1954). It is interesting that similar reductions in social dominance and aggression have been reported following lesions of the orbitofrontal cortex, a region sharing extensive and direct connections with the amygdala (Butter \& Snyder, 1972; Butter, Snyder, \& McDonald, 1970). These data suggest that connections between the orbitofrontal regions and the amygdala may play an important role in regulating complex social behaviors, such as social rank.

The effects of neonatal amygdala damage on the development of a primate social hierarchy have not previously been systematically investigated. Our results are, however, consistent with the initial findings of Thompson et al. (1969), reporting excessive fear behaviors in response to conspecifics following early amygdala damage. Although the amygdala-lesioned subjects studied by Thompson and colleagues were not evaluated in social groups, they did display abnormal subordinate social behaviors later in life (Thompson et al., 1977). It is interesting that early amygdala damage does not lead to heightened fear in all situations. In contrast to the increased fear reported in the present study, amygdala-lesioned subjects actually have been observed to display a lack of fear responses to typically fear-inducing situations or objects (Bauman et al., 2004a; Prather et al., 2001; Thompson et al., 1969). Collectively, these studies indicate that early damage to the amygdala disrupts the ability to correctly evaluate potential 
danger and coordinate an appropriate fear response. The present study further suggests that early amygdala damage profoundly affects the ability to obtain high rank within a naturalistic social hierarchy, possibly through disruption of species-typical fearful and aggressive responses.

Previous studies have suggested that neonatal damage to the hippocampus results in minor disturbances in social interactions during infancy (Bachevalier, 1994), which become more pronounced in adulthood (Bachevalier, Alvarado, \& Malkova, 1999; Beauregard, Malkova, \& Bachevalier, 1995). In contrast, the hippocampus-lesioned subjects in the present study have been indistinguishable from controls on the majority of behavioral assays that have been used thus far (Bauman et al., 2004a, 2004b). It is important to note, however, that the hippocampus-lesioned subjects differed from controls on several measures of food access during the dominance task (i.e., reach order, duration of proximity to the container, and number of reaches in the first minute). We would suggest that it is the hyperactivity previously reported in the hippocampus-lesioned subjects (Bauman et al., 2004a) that may be responsible for the differences in these measures (i.e., the hippocampus-lesioned subjects were observed to constantly move in and out around the food container, which would impact such measures as duration of proximity, number of reaches, and possibly reach order). Moreover, the hippocampus-lesioned subjects did not differ from control subjects on other measures of food access that are not as sensitive to interference from hyperactivity (i.e., latency to first approach the container, latency to reach into the container, frequency of proximity to the container in the first minute) or on any of our behavioral indices of social dominance (i.e., linear rank and use of species-typical fear and aggressive social signals). Of course, we cannot completely rule out the possibility that the hippocampus-lesioned subjects displayed subtle behavioral deficits that have eluded our quantitative observations but were nonetheless detected by conspecifics. Such behavioral changes may have also contributed to the minor differences between control and hippocampus-lesioned subjects reported in the present study.

\section{Conclusion}

Amygdala-lesioned subjects ranked lower than control and hippocampus-lesioned subjects on all behavioral indices of social dominance during access to a limited preferred food. The amygdala-lesioned subjects also showed pronounced differences in the use of species-typical signals associated with fear and aggression during this test (i.e., excessive fear behaviors and diminished aggressive behaviors). Control and hippocampuslesioned subjects were willing to interact with other members of their groups, actively competed for food, and displayed an appropriate combination of both aggressive and submissive social signals. The amygdala-lesioned subjects, in contrast, responded to the food competition by deferring to other group members, more frequently giving expressions of fear, and waiting until they could eventually gain undisputed access to the food after the active competition had abated. These findings have led us to propose that an inability to correctly regulate fearful and aggressive responses may be causally related to the low rank of the amygdala-lesioned subjects.

\section{References}

Altmann, S. (1967). The structure of primate social communication. In S Altmann (Ed.), Social communication among primates (pp. 325-362). Chicago: University of Chicago Press.

Bachevalier, J. (1994). Medial temporal lobe structures and autism: A review of clinical and experimental findings. Neuropsychologia, 32, 627-648.

Bachevalier, J., Alvarado, M. C., \& Malkova, L. (1999). Memory and socioemotional behavior in monkeys after hippocampal damage incurred in infancy or in adulthood. Biological Psychiatry, 46, 329-339.

Bastian, M. L., Sponberg, A. C., Suomi, S. J., \& Higley, J. D. (2003). Long-term effects of infant rearing condition on the acquisition of dominance rank in juvenile and adult rhesus macaques (Macaca mulatta). Developmental Psychobiology, 42, 44-51.

Bauman, M. D., Lavenex, P., Mason, W. A., Capitanio, J. P., \& Amaral, D. G. (2004a). The development of mother-infant interactions after neonatal amygdala lesions in rhesus monkeys. Journal of Neuroscience, 24, 711-721.

Bauman, M. D., Lavenex, P., Mason, W. A., Capitanio, J. P., \& Amaral, D. G. (2004b). The development of social behavior following neonatal amygdala lesions in rhesus monkeys. Journal of Cognitive Neuroscience, 16, 1388-1411.

Beauregard, M., Malkova, L., \& Bachevalier, J. (1995). Stereotypies and loss of social affiliation after early hippocampectomy in primates. NeuroReport, 6, 2521-2526.

Belzung, C., \& Anderson, J. R. (1986). Social rank and responses to feeding competition in rhesus monkeys. Behavioural Processes, 12, 307-316.

Bernstein, I. S. (1981). Dominance: The baby and the bathwater. Behavioral and Brain Sciences, 4, 419-457.

Bernstein, I., \& Ehardt, C. (1985). Age-sex differences in the expression of agonistic behaviors in rhesus monkeys (Macaca mulatta) groups. Journal of Comparative Psychology, 2, 115-132.

Bernstein, I. S., \& Mason, W. A. (1963). Group formation by rhesus monkeys. Animal Behaviour, 11, 28-31.

Butter, C. M., \& Snyder, D. R. (1972). Alterations in aversive and aggressive behaviors following orbital frontal lesions in rhesus monkeys. Acta Neurobiologiae Experimentalis, 32, 525-565.

Butter, C. M., Snyder, D. R., \& McDonald, J. A. (1970). Effects of orbital frontal lesions on aversive and aggressive behaviors in rhesus monkeys. Journal of Comparative and Physiological Psychology, 72, 132-144.

Cheney, D., Seyfarth, R., \& Smuts, B. (1986, December 12). Social relationships and social cognition in nonhuman primates. Science, 234, 1361-1366.

de Waal, F. B. M. (1993). Co-development of dominance relations and affiliative bonds in rhesus monkeys. In M. E. Pereira \& L. A. Fairbanks (Eds.), Juvenile primates: Life history, development, and behavior (pp. 259-270). New York: Oxford University Press.

Drickamer, L. C. (1975). Quantitative observation of behavior in freeranging Macaca mulatta: Methodology and aggression. Behaviour, 55, 209-236.

Emery, N. J., Capitanio, J. P., Mason, W. A., Machado, C. J., Mendoza, S. P., \& Amaral, D. G. (2001). The effects of bilateral lesions of the amygdala on dyadic social interactions in rhesus monkeys (Macaca mulatta). Behavioral Neuroscience, 115, 515-544.

Higley, J. D., King, S. T., Jr., Hasert, M. F., Champoux, M., Suomi, S. J., \& Linnoila, M. (1996). Stability of interindividual differences in serotonin function and its relationship to severe aggression and competent social behavior in rhesus macaque females. Neuropsychopharmacology, 14, 67-76.

Higley, J. D., Mehlman, P. T., Poland, R. E., Taub, D. M., Vickers, J., Suomi, S. J., \& Linnoila, M. (1996). CSF testosterone and 5-HIAA correlate with different types of aggressive behaviors. Biological Psychiatry, 40, 1067-1082. 
Hinde, R. A., \& Spencer-Booth, Y. (1967). The behaviour of socially living rhesus monkeys in their first two and a half years. Animal Behaviour, 15, 169-196.

Izquierdo, A., Suda, R. K., \& Murray, E. A. (2005). Comparison of the effects of bilateral orbital prefrontal cortex lesions and amygdala lesions on emotional responses in rhesus monkeys. Journal of Neuroscience, 25, $8534-8542$.

Janus, M. (1992). Interplay between various aspects in social relationships: Dominance, agonistic help, and affiliation. American Journal of Primatology, 26, 291-308.

Kalin, N. H., Shelton, S. E., Davidson, R. J., \& Kelley, A. E. (2001). The primate amygdala mediates acute fear but not the behavioral and physiological components of anxious temperament. Journal of Neuroscience, 21, 2067-2074.

Kling, A. (1972). Effects of amygdalectomy on socio-affective behavior in non-human primates. In B. E. Eleftheriou (Ed.), Neurobiology of the amygdala (pp. 511-536). New York: Plenum Press.

Kling, A. S. (1992). The amygdala and social behavior. In J. P. Aggleton (Ed.), The amygdala: Neurobiological aspects of emotion, memory, and dysfunction (pp. 353-378). New York: Wiley-Liss.

Kling, A. S., \& Cornell, R. (1971). Amygdalectomy and social behavior in the caged stump-tailed macaque (Macaca speciosa). Folia Primatologica, 14, 190-208.

Mason, W. A. (1961). The effects of social restriction on the behavior of rhesus monkeys: III. Dominance tests. Journal of Comparative and Physiological Psychology, 54, 694-699.

Mason, W. A. (1993). The nature of social conflict: A psycho-ethological perspective. In W. A. Mason \& S. P. Mendoza (Eds.), Primate social conflict (pp. 13-47). Albany: State University of New York Press.

Missakian, E. A. (1972). Genealogical and cross-genealogical dominance relations in a group of free ranging rhesus monkeys (Macaca mulatta) on Cayo Santiago. Primates, 13, 169-180.

Noldus, L. P. (1991). The observer: A software system for collection and analysis of observational data. Behavior Research Methods, Instruments \& Computers, 23, 415-429.

Plotnik, R. (1968). Changes in social behavior of squirrel monkeys after anterior temporal lobectomy. Journal of Comparative and Physiological Psychology, 66, 369-377.
Prather, M. D., Lavenex, P., Mauldin-Jourdain, M. L., Mason, W. A., Capitanio, J. P., Mendoza, S. P., \& Amaral, D. G. (2001). Increased social fear and decreased fear of objects in monkeys with neonatal amygdala lesions. Neuroscience, 106, 653-658.

Reader, S. M., \& Laland, K. N. (2002). Social intelligence, innovation, and enhanced brain size in primates. Proceedings of the National Academy of Sciences, USA, 99, 4436-4441.

Rosvold, H. E., Mirsky, A. F., \& Pribram, K. H. (1954). Influence of amygdalectomy on social behavior in monkeys. Journal of Comparative and Physiological Psychology, 47, 173-178.

Sade, D. S. (1967). Determinants of dominance in a group of free-ranging rhesus monkeys. In S. A. Altmann (Ed.), Social communication among primates (pp. 99-114). Chicago: University of Chicago Press.

Southwick, C. H. (1967). An experimental study of intragroup agonistic behavior in rhesus monkeys (Macaca mulatta). Behaviour, 28, 182-209.

Thierry, B. (1985). Patterns of agonistic interactions in three species of macaque (Macaca mulatta, M. fascicularis, M. tonkeana). Aggressive Behavior, 11, 223-233.

Thierry, B. (1990). Feedback loop between kinship and dominance: The macaque model. Journal of Theoretical Biology, 145, 511-522.

Thompson, C. I., Bergland, R. M., \& Towfighi, J. T. (1977). Social and nonsocial behaviors of adult rhesus monkeys after amygdalectomy in infancy or adulthood. Journal of Comparative and Physiological Psychology, 91, 533-548.

Thompson, C. I., Schwartzbaum, J. S., \& Harlow, H. F. (1969). Development of social fear after amygdalectomy in infant rhesus monkeys. Physiology \& Behavior, 4, 249-254.

Weiskrantz, L. (1956). Behavioral changes associated with ablation of the amygdaloid complex in monkeys. Journal of Comparative and Physiological Psychology, 49, 381-391.

Zola-Morgan, S., Squire, L. R., Alvarez-Royo, P., \& Clower, R. P. (1991). Independence of memory functions and emotional behavior: Separate contributions of the hippocampal formation and the amygdala. Hippocampus, 1, 207-220. 